To appear in Dynamical Systems: An International Journal

Vol. 00, No. 00, Month 20XX, 1-21

\title{
Microbial metabolism and growth under conditions of starvation modelled as the sliding mode of a differential inclusion
}

\author{
O. A. $\mathrm{Nev}^{\mathrm{a}}$ and H. A. van den Berg ${ }^{\mathrm{a} *}$ \\ ${ }^{a}$ University of Warwick, Coventry, CV4 7AL UK
}

(Received 00 Month 20XX; final version received 00 Month 20XX)

\begin{abstract}
We consider a model of bacterial growth with variable internal stores, extended with adaptive resource allocation and investigate the behaviour of this model under conditions of starvation, i.e. severe nutrient shortage, treating the behaviour under the starvation regime in terms of a differential inclusion and derive Filippov solutions. This Filippov sliding mode representation appears to be a simple but sound qualitative description of metabolic 'shut down' in response to starvation. We discuss a natural connection between biologically motivated modelling approaches to metabolic 'shut down' and numerical regularisation techniques to approximate Filippov solutions.
\end{abstract}

Keywords: piecewise smooth systems of ordinary differential equations; Filippov solutions; sliding modes; microbial growth and metabolism; starvation response; metabolic shut down

MCS/CCS/AMS Classification/CR Category numbers: 34A60, 92B05

\section{Introduction}

Systems of ordinary differential equations (ODEs) with discontinuous right-hand sides are called piecewise smooth ( $P W S$ ) systems [1]. The state space of such systems is divided into regions, in each of which the vector field is smooth. The boundaries between these regions are called switching surfaces or surfaces of discontinuity.

Filippov and Utkin pioneered the mathematical treatment of PWS systems [2,3], which have subsequently proven to be invaluable in engineering and biological applications. In particular, PWS systems arise in control theory [3, 4] and the study of complementary and hybrid systems [5, 6], as well as in several other applications [7-9]. In biology, genetic regulatory networks have profitably been treated as PWS systems [10-14]. In a recent paper on the modelling of such networks, Gouze and Sari [15] applied the Filippov theory [2] to investigate the behaviour of the system on its surfaces of discontinuity. This approach, which is also widely used in control theory, involves an extension of the discontinuous system into a differential inclusion [2]. The solutions of this extended system on the surfaces of discontinuity are called sliding modes [2].

Here, we analyse a PWS system that describes bacterial growth and growth cessation under conditions of nutrient shortage (starvation). The model we study is a variableinternal-stores (VIS) model [16-18] accounting for changes in cellular composition under varying conditions of nutrient availability [19]. Our VIS-plus-reallocation model [20], accounts explicitly for the regulation of the allocation of molecular building blocks among

\footnotetext{
*Corresponding author. Email: hugo@maths.warwick.ac.uk
} 
various types of catalytic machinery [21, 22]. The changes in the rates at which these building blocks are incorporated into machinery proteins correspond to changes in gene expression and allow the organism to respond to environmental variations [23].

We investigate the behaviour of the VIS-with-reallocation model when at least one of the internal stores in the cell is approaching the point of complete depletion. To this end, we apply the Filippov theory and derive the resulting dynamics in terms of sliding modes. These constitute the mathematical counterpart to the biological phenomenon of metabolic "shut down," the organism's response to nutrient shortage whereby the rate of metabolism slows down or even comes to a complete stop [19, 24, 25]. We argue that sliding modes provide a versatile and convenient formalism to represent such transitory behaviours in metabolic systems, where this might otherwise require a more cumbersome dynamical system representation, e.g. of higher dimension.

The paper is organised as follows. In Section 2 we discuss the Filippov theory, establish notation, and review the results required for our application to the problem of metabolic "shut down." In Section 3 we review the VIS-with-reallocation model. In Section 4 we formulate the model as a PWS system and apply the Filippov theory [2] to find the solution of the discontinuous system on the surface of discontinuity. Finally, we consider regularisations in Section 5.

\section{Filippov solutions of differential equations with discontinuities}

In this section we briefly review Filippov's approach to defining a solution for PWS systems [2]. We restrict ourselves to the simple case where the state space $\mathbb{R}^{n}$ is divided into two regions, $S^{+}$and $S^{-}$, in each of which the vector field is smooth, and which are separated by a boundary $\Sigma$, so that $\mathbb{R}^{n}=S^{+} \cup \Sigma \cup S^{-}$. We consider a point in state space $\mathbf{X} \in \mathbb{R}^{n}$ with discontinuous dynamics:

$$
\dot{\mathbf{X}}=f(\mathbf{X})=\left\{\begin{array}{ll}
f^{+}(\mathbf{X}), & \text { if } \mathbf{X} \in S^{+} \\
f^{-}(\mathbf{X}), & \text { if } \mathbf{X} \in S^{-},
\end{array} \quad \mathbf{X}(0)=\mathbf{X}_{0} \in \mathbb{R}^{n},\right.
$$

where the dot indicates differentiation with respect to time and the dynamics $f(\cdot)$ is defined and smooth on each of the subspaces $S^{+}$and $S^{-}$, but is not defined on the boundary $\Sigma$. In particular, $f^{+}(\cdot)$ is assumed to be at least once continuously differentiable on $S^{+} \cup \Sigma$ but not defined on $S^{-}$, whereas $f^{-}(\cdot)$ is assumed to be at least once continuously differentiable on $S^{-} \cup \Sigma$ but not defined on $S^{+}$.

Since the right-hand side of system (1) is not defined on the boundary (surface of discontinuity) $\Sigma$, an extension of the vector field is required. To this end, the Filippov convex method [2] extends the discontinuous system to a convex differential inclusion:

$$
\dot{\mathbf{X}} \in F(\mathbf{X})= \begin{cases}f^{+}(\mathbf{X}), & \text { if } \mathbf{X} \in S^{+} \\ f^{F}(\mathbf{X}), & \text { if } \mathbf{X} \in \Sigma \\ f^{-}(\mathbf{X}), & \text { if } \mathbf{X} \in S^{-}\end{cases}
$$

where $f^{F}(\mathbf{X}) \in \mathbb{R}^{n}$ is a minimal closed convex set containing $f^{+}(\mathbf{X})$ and $f^{-}(\mathbf{X})$ :

$$
f^{F}(\mathbf{X})=(1-\lambda) f^{-}(\mathbf{X})+\lambda f^{+}(\mathbf{X}),
$$

where $\lambda \in[0,1]$ is a convex "mixing" coefficient. 
The differential inclusion can be regarded as a variable structure system [3, 4] with control input $u(\mathbf{X}) \in[0,1]$ by defining

$$
f(\mathbf{X}, u)=(1-u) f^{-}(\mathbf{X})+u f^{+}(\mathbf{X})
$$

where $u \equiv u^{+}=0$ if $\mathbf{X} \in S^{-}, u \equiv u^{-}=1$ if $\mathbf{X} \in S^{+}$, and $u=\lambda$ if $\mathbf{X} \in \Sigma$. On this approach, $f$ is a regular smooth dynamics that acquires its sliding mode characteristics through the choice of the control $u$, i.e., for suitable $u$, we have $f \equiv F$.

\subsection{Construction and nature of Filippov solutions}

Let us formally state what we mean by a solution in the sense of Filippov [2].

Definition 2.1 (solution in the sense of Filippov): An absolutely continuous function $\mathbf{X}(t)$ is said to be a solution of (1) in the sense of Filippov, or Filippov solution, if for almost all $t$,

$$
\dot{\mathbf{X}} \in F(\mathbf{X}(t))
$$

where $F(\mathbf{X}(t))$ is as described by the differential inclusion (2).

Such a solution is guaranteed to exist by the following theorem [26].

Theorem 2.2 (existence of Filippov solution): Provided that $F(\mathbf{X})$ is upper semicontinuous and has a compact, convex image, the solution of the Filippov differential inclusion (2) always exists.

Remark 2.3: By construction, $F(\cdot)$ as stated in (2) satisfies the conditions of Theorem 2.2 .

Specification of the Filippov solution is facilitated by introducing a scalar switching function $h: \mathbb{R}^{n} \rightarrow \mathbb{R}$ which is at least twice continuously differentiable, and is such that $h(\mathbf{X})$ has a nonzero gradient on the surface of discontinuity $\Sigma$, with

$$
\begin{gathered}
S^{+}=\left\{\mathbf{X} \in \mathbb{R}^{n}: h(\mathbf{X})>0\right\}, \quad \Sigma=\left\{\mathbf{X} \in \mathbb{R}^{n}: h(\mathbf{X})=0\right\}, \\
S^{-}=\left\{\mathbf{X} \in \mathbb{R}^{n}: h(\mathbf{X})<0\right\} .
\end{gathered}
$$

Let us consider a solution of eqn (1) starting in $S^{+}$and reaching $\Sigma$ in a finite time. At this point, the trajectory may either immediately exit the boundary and enter one of the subspaces, or stay on the boundary. This depends on the directional derivatives $(\nabla h(\mathbf{X}))^{T} f^{+}(\mathbf{X})$ and $(\nabla h(\mathbf{X}))^{T} f^{-}(\mathbf{X})$. The trajectory may either cross $\Sigma$ or enter it in a sliding mode. A crossing occurs when

$$
(\nabla h(\mathbf{X}))^{T} f^{+}(\mathbf{X})(\nabla h(\mathbf{X}))^{T} f^{-}(\mathbf{X})>0
$$

is satisfied for $\mathbf{X} \in \Sigma$. In this case the trajectory leaves the surface of discontinuity and enters $S^{+}$if and only if $(\nabla h(\mathbf{X}))^{T} f^{-}(\mathbf{X})>0$ (otherwise it enters $S^{-}$). The system enters $\Sigma$ in a slide when

$$
(\nabla h(\mathbf{X}))^{T} f^{+}(\mathbf{X})(\nabla h(\mathbf{X}))^{T} f^{-}(\mathbf{X})<0
$$


for $\mathbf{X} \in \Sigma$. This sliding mode can be either attracting or repulsive. The former requires

$$
(\nabla h(\mathbf{X}))^{T} f^{+}(\mathbf{X})<0 \quad \text { and } \quad(\nabla h(\mathbf{X}))^{T} f^{-}(\mathbf{X})>0 .
$$

In this case the trajectory continues on the boundary with the vector field $f^{F}(\mathbf{X})$ defined by eqn (3) with the following specification for $\lambda$ :

$$
\lambda=\frac{(\nabla h(\mathbf{X}))^{T} f^{-}(\mathbf{X})}{(\nabla h(\mathbf{X}))^{T} f^{-}(\mathbf{X})-(\nabla h(\mathbf{X}))^{T} f^{+}(\mathbf{X})} .
$$

The attracting sliding mode solution thus defined exists and is unique [2]. On the other hand, when

$$
(\nabla h(\mathbf{X}))^{T} f^{+}(\mathbf{X})>0 \quad \text { and } \quad(\nabla h(\mathbf{X}))^{T} f^{-}(\mathbf{X})<0,
$$

the sliding mode is said to be repulsive and several further subcases arise which we need not discuss for the purposes of the present paper.

\subsection{First-order exit conditions}

As long as the attracting sliding mode conditions (6) are met, the system will remain confined to the switching surface $\Sigma$, either stationarily or moving along a trajectory contained in $\Sigma$. Either these conditions will remain satisfied as $t \rightarrow \infty$, or there is an earliest point in time after entering $\Sigma$ when the conditions are no longer satisfied. In the latter case, the system may exit $\Sigma$, but this is by no means a necessity; the outcome depends on a set of criteria called the exit conditions, which have been studied in detail by Dieci and Lopez [1], who provided first-order exit conditions and discussed higher-order exit conditions which must be invoked when the first-order conditions are inconclusive. We restrict our discussion to the first-order exit conditions as they suffice for our purposes. Without loss of generality, we write $t=0$ for the earliest point in time, after entry of $\Sigma$, when conditions (6) are violated, and $t=0^{-}$for a point in time immediately before $t=0$. The first-order exit conditions state that the sliding trajectory leaves the surface of discontinuity $\Sigma$ and enters the subspace $S^{+}$along the vector field $f^{+}(\mathbf{X})$ if at $t=0$ the following conditions are satisfied:

$$
\left\{\begin{array}{l}
(\nabla h(\mathbf{X}))^{T} f^{-}(\mathbf{X})>0 \\
(\nabla h(\mathbf{X}))^{T} f^{+}(\mathbf{X})=0 \\
\left.\frac{\partial}{\partial \mathbf{X}} g(\mathbf{X}) \dot{\mathbf{X}}\right|_{t=0^{-}}>0
\end{array}\right.
$$

where $g(\mathbf{X})=(\nabla h(\mathbf{X}))^{T} f^{+}(\mathbf{X})$ and $\mathbf{X} \in \Sigma$. Exit from $\Sigma$ to $S^{-}$is, by symmetry, subject to analogous conditions.

\section{Variable internal store-with-reallocation model}

In this section we present the VIS-with-reallocation model, which is a minor generalisation of the version in a companion paper [20]. Figure 1 shows a schematic diagram of the model, which is based on the standard principles of chemical conservation, expressed by 


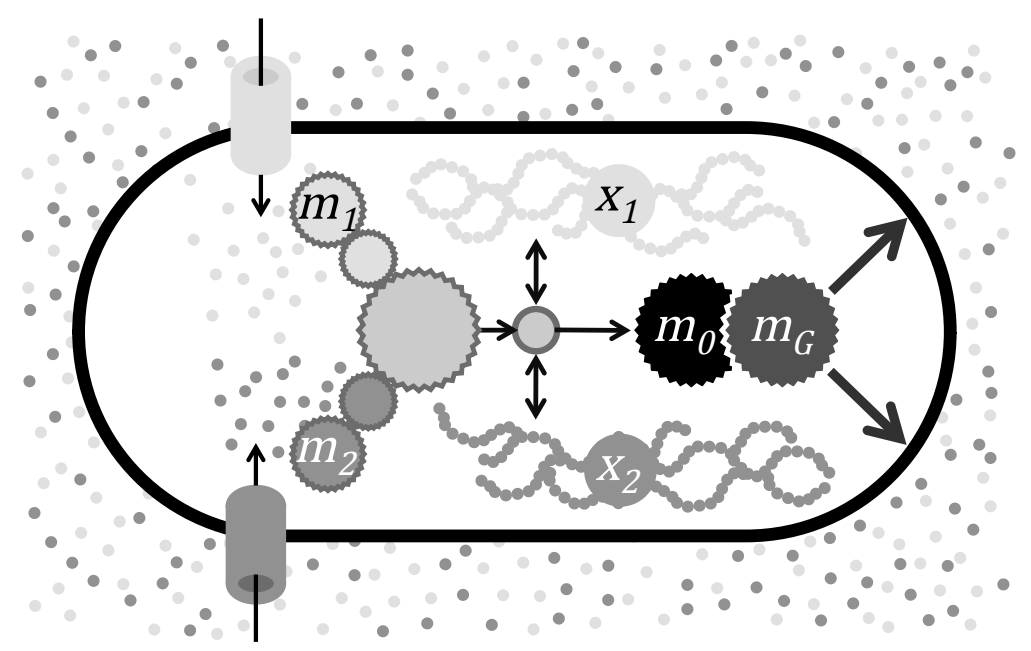

Figure 1. Schematic representation of the model described by system (14) for the case $n=2$. Two types of nutrients are assimilated by dedicated pathways $\left(m_{1}\right.$ and $\left.m_{2}\right)$ that feed into core metabolism from which building blocks are taken to machinery synthesis $\left(m_{0}\right)$ and growth $\left(m_{G}\right)$. Core metabolism also exchanges molecular building blocks with reserves $\left(x_{1}\right.$ and $\left.x_{2}\right)$.

stoichiometric equations detailed in Section 3.1. In addition, the VIS-with-reallocation model comprises so-called regulatory laws that govern the allocation of molecular building blocks among various types of catalytic molecular machinery; these are detailed in Section 3.2. A novel extension, accounting for nutrient starvation, is presented in Section 3.3 .

\subsection{Stoichiometric equations}

The model is based on the partitioning of a bacterial cell into three kinds of components: reserve polymers, machinery, and a structural component. The components each satisfy elemental homeostasis and thus have a fixed empirical formula which in most cases is normalised relative to carbon (C), giving C-molar quantities for each.

The reserve components serve as nutrient repositories and are mobilised by the cell to replenish the central pools of core metabolites. Many reserves (e.g. lipids, poly- $\beta$ hydroxybutyrate, polysaccharides, storage proteins) are expressible in terms of C-moles, whereas others (e.g. sulphur globules and polyphosphate inclusions) contain no carbon and have to be expressed in terms of their dominant element: if the primary element of the reserve of type $j$ is $\mathrm{X}_{j}$, the $\mathrm{X}_{j}$-molar amount is used to quantify reserve $j$ and this amount is denoted as $X_{j}$ for $j \in\{1, \ldots, n\}$.

Molecular machinery is divided into $n+2$ components, where $n$ is the number of different chemical species of essential nutrients that are accounted for in the model. Component 0 is the molecular machinery required to synthesise de novo machinery. Components 1 through $n$ are machinery dedicated to the assimilation of the corresponding nutrients from the environment, and to the conversion of these nutrients into core metabolites. The last component $n+1$, given the special subscript $G$, is the machinery devoted to growth, which includes the synthesis of the cell envelope and the duplication of the genome. The C-molar amount of the molecular machinery of type $i$ is denoted by $M_{i}$ for $i \in\{0,1, \ldots, n, G\}$.

The structural component, whose C-molar amount will be denoted as $W$, comprises 
everything not covered by the first two categories. It includes the cell envelope, genetic material, and the small molecules of metabolism, which are maintained at nominal cellular concentrations via mechanisms that are not explicitly represented in this model.

Since all types of molecular machinery are synthesised by the machinery of type 0 , we have:

$$
\dot{M}_{i}=\alpha_{i} M_{0} \widetilde{\phi}_{i}, \quad i \in\{0,1, \ldots, n, G\} .
$$

The stoichiometric coefficient $\widetilde{\phi}_{i}$ in eqn (9) shows the rate of production of the machinery of type $i$; the allocation coefficient $\alpha_{i}$, discussed further in Section 3.2, defines the portion of zero-machinery that is dedicated for synthesis of the corresponding type $i$ of machinery. The parameters $\alpha_{i}$ satisfy $\sum_{i \in\{0,1, \ldots, n, G\}} \alpha_{i}=1$. Each reserve component is subject to uptake and expenditure fluxes:

$$
\begin{aligned}
\dot{X}_{j}=\sum_{i=1}^{n} \widetilde{\psi}_{j i} M_{i}-\widetilde{\sigma}_{j W} \dot{W}-M_{0} \sum_{i \in\{0,1, \ldots, n, G\}} \widetilde{\sigma}_{j i} \alpha_{i} \widetilde{\phi}_{i}-\widetilde{c}_{j} W, \\
i \in\{0,1, \ldots, n, G\}, j \in\{1, \ldots, n\},
\end{aligned}
$$

where the first term represents an aggregated gain of reserve $j$ from the influx through 1 to $n$ types of assimilatory machinery, and the three remaining terms represent expenditures on, respectively, structural component growth, machinery production, and maintenance $[27,28]$. Accordingly, the stoichiometric parameters have the following biological interpretation: $\widetilde{\psi}_{j i}$ is the gain of reserve $j$ per unit machinery of type $i$; $\widetilde{\sigma}_{j W}$ is the loss of reserve $j$ per unit increase of $W$; $\widetilde{\sigma}_{j i}$ is the loss of reserve $j$ per unit synthesis of machinery of type $i$; $\widetilde{c}_{j}$ is the loss of reserve $j$ that is being catabolised per unit of structural biomass to maintain cellular integrity (even in the absence of growth and synthesis of machinery). The growth machinery is dedicated to the construction of the structural component:

$$
\dot{W}=\widetilde{\psi}_{W} M_{G},
$$

where $\widetilde{\psi}_{W}$ is the rate of production of the structural component. The tilde over the stoichiometric parameters signifies that they are dimensional. Choosing suitable parameters as natural units, we can render the equations nondimensional and we will let the corresponding symbols without tilde denote dimensionless (scaled) quantities. In particular, adopting $\widetilde{\phi}_{0}^{-1}$ as a unit of time, we define scaled state variables as follows:

$$
m_{i}=\frac{M_{i} \widetilde{\phi}_{0}}{W \widetilde{m}_{i}} ; \quad x_{j}=\frac{X_{j}}{W \widetilde{\sigma}_{j W}},
$$

where $\widehat{m}$ is a scaling parameter for $M_{0} / W$. Scaled stoichiometric parameters are defined as follows:

$$
\psi_{j i}=\frac{\widetilde{\psi}_{j i} \widetilde{\phi}_{i} \widehat{m}}{\widetilde{\sigma}_{j W} \widetilde{\phi}_{0}^{2}} ; \quad \psi_{W}=\frac{\widetilde{\psi}_{W} \widetilde{\phi}_{G} \widehat{m}}{\widetilde{\phi}_{0}^{2}} ; \quad \sigma_{j i}=\frac{\widetilde{\sigma}_{j i} \widetilde{\phi}_{i} \widehat{m}}{\widetilde{\sigma}_{j W} \widetilde{\phi}_{0}} ; \quad c_{j}=\frac{\widetilde{c}_{j}}{\widetilde{\sigma}_{j W} \widetilde{\phi}_{0}} .
$$

This scaling gives $\left(W \widetilde{\phi}_{0}\right)^{-1} \dot{W}=\psi_{W} m_{G}$ for the specific growth rate which microbial physiologists usually denoted by $\mu[24,29]$. For the sake of simplicity, we assume that 
for every reserve $j$ we have the same expenditure coefficient for each type of molecular machinery $i: \sigma_{j i}=\sigma_{j}$, which is reasonable when different types of machinery are biochemically similar, i.e. they require similar relative amounts of reserves for synthesis.

The final scaled system of differential equations takes on the following form:

$$
\begin{cases}\dot{x}_{j}=\psi_{j} m_{j}-\mu\left(1+x_{j}\right)-m_{0} \sigma_{j}-c_{j} & \text { for } j \in\{1, \ldots, n\} \\ \dot{m}_{i}=\alpha_{i} m_{0}-\mu m_{i} & \text { for } i \in\{0,1, \ldots, n, G\},\end{cases}
$$

where the $\psi_{j}$ denote stoichiometric coefficients whose derivation is detailed in a companion paper [20].

\subsection{Constitutive relationships: regulatory laws}

The allocation of molecular building blocks to the various types of catalytic machinery is expressed by the coefficients $\alpha_{0}, \alpha_{1}, \ldots, \alpha_{n}, \alpha_{G}$ which can be interpreted, in somewhat loose terms, as the fraction of time a ribosome will typically spend on each of these destinations. To close the equations, we require expressions for these coefficients in terms of the other model variables. Accordingly, we treat the allocation coefficients as functions of the internal state variables and/or environmental parameters. In keeping with the idea of "ribosome time" we use the following expression:

$$
\alpha_{i}=\widetilde{r}_{i} /\left(\widetilde{r}_{0}+\widetilde{r}_{1}+\cdots+\widetilde{r}_{n}+\widetilde{r}_{G}\right),
$$

where $\widetilde{r}_{i}$ is proportional to the concentration of translationally active mRNA for machinery of type $i$. For the sake of simplicity, we assume that $\widetilde{r}_{0}$ is a constant and use the scaling $r_{i}=\widetilde{r}_{i} / \widetilde{r}_{0}$ giving

$$
\alpha_{i}=r_{i}\left(1+r_{1}+\cdots+r_{n}+r_{G}\right)^{-1} .
$$

We assume that the $r_{j}$ are decreasing sigmoid functions of corresponding reserve densities $x_{j}$, as follows:

$$
r_{j}=\widehat{r}_{j}\left(1+\exp \left\{\vartheta_{j}\left(x_{j}-\xi_{j}\right)\right\}\right)^{-1}
$$

where $\widehat{r}_{j}, \vartheta_{j}$, and $\xi_{j}$ are positive shape parameters. This arrangement creates a negative feedback loop in which the amount of machinery synthesised is "counterskewed" to ambient nutrient availabilities and thus the reserve densities are regulated toward the midpoint $\xi_{j}$ of the sigmoid curve.

Finally, $r_{G}$ is an increasing function of $m_{0}$, which creates another feedback loop that adjusts the growth rate to overall resource availability:

$$
r_{G}\left[m_{0}\right]= \begin{cases}0, & \text { if } m_{0} \leq 1-\delta \\ r_{G, \max } / 2+K\left(m_{0}-1\right), & \text { if } 1-\delta<m_{0} \leq 1+\delta \\ r_{G, \max }, & \text { if } m_{0}>1+\delta\end{cases}
$$

where $K$ is a positive parameter which can be estimated from the observed relationship between the cell's RNA content and relative growth rate [29] and $\delta=r_{G, \max } /(2 K)$.

The coefficients $\psi_{j}$ are dependent on environmental conditions and should generally be regarded as time-varying. Specifically, $\psi_{j}$ is assumed to be a monotone increasing function 
of the ambient concentration of the $j$ th essential nutrient. Under constant environmental conditions, we have $\dot{\psi}_{j}=0$ for all $j$ and the system has an equilibrium point which is unique and stable (the proof provided earlier assumed $c_{j} \equiv 0 \forall j$, but all its steps go through substantially unaltered under the notational change $\left.\sigma_{j} \rightarrow \sigma_{j}+c_{j} / m_{0}\right)$.

\subsection{Starvation and metabolic "shut down"}

Starvation refers in general to a restricted environmental availability of one or more essential nutrients, or even the complete absence of one or several of them. However, in those cases where the cell has accumulated a substantial amount of reserves, it may be able to bridge periods of low or zero ambient nutrient concentration by mobilising these results. How long the cell is able to do this depends on the duration of the "famine" and also on the levels of reserves it has built up; the latter depends in turn on the regulatory laws.

If the "famine" endures for a sufficiently long period of time, one or more reserves will be depleted (i.e. one or more $x_{j}$ will approach the value 0) and the cell will have to respond in some manner. Here we will use the general term "shut down" to indicate a slowing down or even complete cessation of metabolic rates; in reality microbial responses to nutrient stress are extraordinarily diverse as well as species-dependent [19] and we proffer the present treatment as a minimal general model of such responses.

The model as stated is not applicable to the shut-down situation, inasmuch as the expenditure fluxes are not donor-controlled. At a microscopic level, the rate of a biochemical reaction depends on the chemical activities of the reactants and the products and thus it may appear that the model as stated in Section 3.1 is fundamentally flawed. However, at the macro-chemical level, this model structure can be justified by appealing to the homeostasis of the structural component. In particular, this homeostasis includes a pool of small core metabolites which are the point of departure for all anabolic pathways [24]. As long as these metabolites are maintained at nominal levels, the expenditures behave as if demand-driven. It is precisely this assumption that breaks down when $x_{j} \downarrow 0$ for one or more reserve $j$.

Accordingly, we specify that the dynamics as stated in Section 3.1 is restricted to the case where all reserve densities are non-negative, and we require an additional set of equations to describe the dynamics when $x_{j}<0$ for one or more $j$. From a biological perspective, negative reserve densities cannot exist, and they are to be interpreted as violations of the compositional homeostasis of the structural component. More specifically, negative reserve densities express excursions of one or more core metabolites below their nominal concentrations. We shall assume that the cell responds to this situation by shrinking at a relative rate $-\nu$ (where $\nu$ is a positive constant) to provide the materials to restore structural homeostasis. At the same time, we assume that anabolic and maintenance expenditures are stopped. These assumptions lead to the following ODEs (in scaled form):

$$
\left\{\begin{array}{ll}
\dot{x}_{j}=\psi_{j} m_{j}+\nu\left(1+x_{j}\right) & \text { for } j \in\{1, \ldots, n\} \\
\dot{m}_{i}=\nu m_{i} & \text { for } i \in\{0,1, \ldots, n, G\}
\end{array} .\right.
$$

This extension is valid only in the near vicinity of the boundary of the region where the original model is valid. The pools of core metabolites, while crucial to the cell's physiology, are quantitatively minor. Therefore, only minute negative excursions of $x_{j}$ can plausibly be interpreted as depletions of these pools. Moreover, the cell's physical scope for de- 
growth (structural shrinkage) is likely to be severely limited, given the chemical structure of the outer envelope $[24,30]$. However, in anticipation of the Filippov solutions, we only require valid dynamics in close vicinity to the boundary which is the locus of $\prod_{j=1}^{n} x_{j}=0$.

\section{Sliding modes of the extended VIS-with-reallocation model}

The previous section presented a PWS system of the following general form

$$
\dot{\mathbf{X}}=f(\mathbf{X}), \quad \mathbf{X}=\left(x_{j} ; m_{i}\right)^{T} \quad \text { for } i \in\{0,1, \ldots, n, G\}, j \in\{1, \ldots, n\},
$$

where superscript $T$ denotes transposition, the dynamics $f(\cdot)$ is specified by two sets of ODEs, eqns (14) and (19), each with its own domain of validity. In this section we investigate the behaviour of this system under conditions of starvation that are sufficiently severe to drive one or more reserve densities down to zero, and show that metabolic "shut down" corresponds to a sliding mode of the PWS system on the boundary defined by

$$
\Sigma=\left\{\mathbf{X}: \prod_{j=1}^{n} x_{j}=0\right\} .
$$

\subsection{Filippov solution for single reserve}

The state space $\mathbb{R}^{4}$ is divided into two regions, $S^{+}$and $S^{-}$, according to the sign of $x_{1}$, which are separated by $\Sigma$, the locus of $x_{1}=0$. For $\mathbf{X} \in S^{+}$we have $f(\mathbf{X})=f^{+}(\mathbf{X})$ where $f^{+}$is defined on $S^{+}$. Letting $\xi_{1}^{+}$denote the dynamics of the reserve density $x_{1}$ and let $\eta_{i}^{+}$denote the dynamics of the molecular machineries $m_{i}$ for $i \in\{0,1, G\}$, we have the following dynamics for $\mathbf{X} \in S^{+}$:

$$
\dot{\mathbf{X}}=f^{+}(\mathbf{X})=\left(\xi_{1}^{+} ; \eta_{i}^{+}\right)^{T}, \quad \mathbf{X}=\left(x_{1} ; m_{i}\right)^{T} \text { for } i \in\{0,1, G\},
$$

where $\xi_{1}^{+}$and $\eta_{i}^{+}$are defined in accordance with system (14), as follows:

$$
\xi_{1}^{+}=\psi_{1} m_{1}-\psi_{W} m_{G}\left(1+x_{1}\right)-\sigma_{1} m_{0}-c_{1}, \quad \eta_{i}^{+}=\alpha_{i} m_{0}-\psi_{W} m_{G} m_{i}
$$

for $i \in\{0,1, G\}$. Similarly, the dynamics for $\mathbf{X} \in S^{-}$is as follows:

$$
\dot{\mathbf{X}}=f^{-}(\mathbf{X})=\left(\xi_{1}^{-} ; \eta_{i}^{-}\right)^{T}, \quad \mathbf{X}=\left(x_{1} ; m_{i}\right)^{T} \text { for } i \in\{0,1, G\},
$$

where $f^{-}$is defined on $S^{-}$, with the following definitions for $\xi_{1}^{-}$and $\eta_{i}^{-}$:

$$
\xi_{1}^{-}=\psi_{1} m_{1}+\nu\left(1+x_{1}\right), \quad \eta_{i}^{-}=\nu m_{i} \quad \text { for } i \in\{0,1, G\}
$$

in accordance with system (19). Combining the vector fields $f^{+}(\mathbf{X})$ and $f^{-}(\mathbf{X})$, we arrive at the following PWS system:

$$
\dot{\mathbf{X}}= \begin{cases}f^{+}(\mathbf{X})=\left(\xi_{1}^{+} ; \eta_{i}^{+}\right)^{T}, & \text { if } \mathbf{X} \in S^{+} \\ f^{-}(\mathbf{X})=\left(\xi_{1}^{-} ; \eta_{i}^{-}\right)^{T}, & \text { if } \mathbf{X} \in S^{-}\end{cases}
$$

Filippov's approach requires that we characterise the surface of discontinuity $\Sigma$ together with the subspaces $S^{+}$and $S^{-}$by means of a suitably chosen scalar smooth 
function $h(\mathbf{X}), \mathbf{X} \in \mathbb{R}^{4}$, such that

$$
S^{+}=\{\mathbf{X}: h(\mathbf{X})>0\}, \quad S^{-}=\{\mathbf{X}: h(\mathbf{X})<0\}, \quad \Sigma=\{\mathbf{X}: h(\mathbf{X})=0\} .
$$

These conditions are satisfied if we set $h(\mathbf{X})=x_{1}$. The Filippov convex method leads us to consider a differential inclusion as stated in eqn (2) with the vector field $f^{F}(\mathbf{X})$ on the boundary $\Sigma$ defined by eqn (3) with convex "mixing" coefficient $\lambda$ as given by eqn (7). In the case of system (24) we find

$$
(\nabla h(\mathbf{X}))^{T} f^{-}(\mathbf{X})=\xi_{1}^{-} ; \quad(\nabla h(\mathbf{X}))^{T} f^{+}(\mathbf{X})=\xi_{1}^{+},
$$

and thus, by eqn (7),

$$
\lambda=\xi_{1}^{-} /\left(\xi_{1}^{-}-\xi_{1}^{+}\right)
$$

which allows us to calculate the boundary dynamics $f^{F}(\cdot)$. In particular, we have

$$
f^{F}\left(x_{1}\right)=(1-\lambda) \xi_{1}^{-}+\lambda \xi_{1}^{+}=\frac{-\xi_{1}^{+}}{\xi_{1}^{-}-\xi_{1}^{+}} \xi_{1}^{-}+\frac{\xi_{1}^{-}}{\xi_{1}^{-}-\xi_{1}^{+}} \xi_{1}^{+}=0
$$

which shows that a sliding mode with $x_{1}(t) \equiv 0$ is feasible. We recall from Section 2 that there are two principal ways for the trajectory to continue. The first is a crossing, subject to condition (5) which here takes on the form

$$
\left(\psi_{1} m_{1}-\psi_{W} m_{G}\left(1+x_{1}\right)-\sigma_{1} m_{0}-c_{1}\right)\left(\psi_{1} m_{1}+\nu\left(1+x_{1}\right)\right)>0 .
$$

Since $\mathbf{X} \in \Sigma$ we have $x_{1}=0$, and thus this crossing condition becomes:

$$
\left(\psi_{1} m_{1}-\psi_{W} m_{G}-\sigma_{1} m_{0}-c_{1}\right)\left(\psi_{1} m_{1}+\nu\right)>0 .
$$

Since $\nu$ is a positive constant and $\psi_{1}$ and $m_{1}$ are nonnegative, the expression in the second pair of brackets is always positive, and the crossing condition can be further simplified to

$$
\psi_{1} m_{1}>\psi_{W} m_{G}+\sigma_{1} m_{0}+c_{1} .
$$

Since $\psi_{1}$ is a monotone increasing function of the ambient concentration of the essential nutrient, this condition will be satisfied when the environmental level of this nutrient is sufficiently high. By contrast, if the nutrient concentration lies below a critical threshold, the crossing condition (25) is not met and the system is prevented from re-entering $S^{+}$. To establish that a sliding mode ensues, we turn to the attracting sliding mode conditions (6). These take on the following form for $\mathbf{X} \in \Sigma$ :

$$
\left\{\begin{array}{l}
\psi_{1} m_{1}+\nu>0 \\
\psi_{1} m_{1}<\psi_{W} m_{G}+\sigma_{1} m_{0}+c_{1}
\end{array}\right.
$$

The first inequality is satisfied as $\psi_{1}$ and $m_{1}$ are nonnegative and $\nu$ is a positive constant. The second inequality is just the opposite of the crossing condition. We may thus conclude that there is a critical value of the nutrient concentration, above which the crossing 
condition is met, and below which the attracting sliding condition is met. To this critical value of the nutrient concentration there corresponds a critical value of $\psi_{1}$ since $\psi_{1}$ is a monotone increasing function of the nutrient concentration. We shall write this critical value as $\psi_{1}^{\star}=\left(\psi_{W} m_{G}+\sigma_{1} m_{0}+c_{1}\right) / m_{1}$.

We anticipate that the system will exit the sliding mode when the ambient conditions improve and $\psi_{1}$ becomes equal to, or exceeds, the critical value $\psi_{1}^{\star}$. To verify this, we employ the first-order exit conditions (Section 2.2). The first condition from system (8) becomes

$$
(\nabla h(\mathbf{X}))^{T} f^{-}(\mathbf{X})=\psi_{1} m_{1}+\nu>0
$$

which is satisfied, and the second condition from (8) takes on the form

$$
(\nabla h(\mathbf{X}))^{T} f^{+}(\mathbf{X})=\psi_{1} m_{1}-\psi_{W} m_{G}-\sigma_{1} m_{0}-c_{1}=0
$$

which is satisfied when $\psi_{1}=\psi_{1}^{\star}$. The third condition requires a bit more care. According to Dieci and Lopez [1], if the first two conditions from (8) are satisfied, we know that

$$
\left.\frac{\partial}{\partial \mathbf{X}} g(\mathbf{X}) \dot{\mathbf{X}}\right|_{t=0^{-}} \geq 0
$$

(where $t=0^{-}$is the last point in time where attracting sliding mode conditions are satisfied) and if equality can be ruled out, the third exit condition in (8) will be satisfied. For this purpose, it suffices to establish strict inequality for at least one component which we choose to be $x_{1}$. We have

$$
\left.\frac{\partial}{\partial x_{1}} g(\mathbf{X}) \dot{x_{1}}\right|_{t=0^{-}}=-\left.\psi_{W} m_{G}\left(\psi_{1} m_{1}-\psi_{W} m_{G}\left(1+x_{1}\right)-\sigma_{1} m_{0}-c_{1}\right)\right|_{t=0^{-}} .
$$

At $t=0^{-}$the trajectory is still on the surface of discontinuity and $x_{1}\left(0^{-}\right)=0$. Hence

$$
\left.\frac{\partial}{\partial x_{1}} g(\mathbf{X}) \dot{x_{1}}\right|_{t=0^{-}}=\psi_{W} m_{G}\left(-\psi_{1} m_{1}+\psi_{W} m_{G}+\sigma_{1} m_{0}+c_{1}\right) \text {. }
$$

The factor $\psi_{W} m_{G}$ is positive because both $\psi_{W}$ and $m_{G}$ are positive, as is the expression in brackets since $\psi_{1}<\psi_{1}^{\star}$ at $t=0^{-}$, which follows as $t=0$ is by definition the first instant in time when $\psi_{1} \geq \psi_{1}^{\star}$ becomes true. We conclude that

$$
\left.\frac{\partial}{\partial x_{1}} g(\mathbf{X}) \dot{x_{1}}\right|_{t=0^{-}}>0
$$

and thereby all first-order exit conditions are satisfied. We conclude that the dynamics "unfreezes" as soon as ambient conditions become sufficiently favourable and the system will re-enter $S^{+}$. Whenever $\mathbf{X}$ attains the boundary $\Sigma$ coming from $S^{+}$, it will follow sliding mode dynamics as long as $\psi_{1}<\psi_{1}^{\star}$ and exit $\Sigma$ to $S^{+}$as soon as $\psi_{1} \geq \psi_{1}^{\star}$. If $\psi_{1}$ is admitted as a state variable, this scenario can be classified as a grazing-sliding bifurcation of codimension $1[31,32]$.

The behaviour of the PWS system to a piece-wise constant forcing function $\psi_{1}(t)$, representing an alternation of "feast" and "famine" conditions, is shown in Fig. 2. During the "famines" the reserve runs down and the system enters a sliding mode with $x_{1} \equiv 0$ from which it exits as soon as conditions improve. 

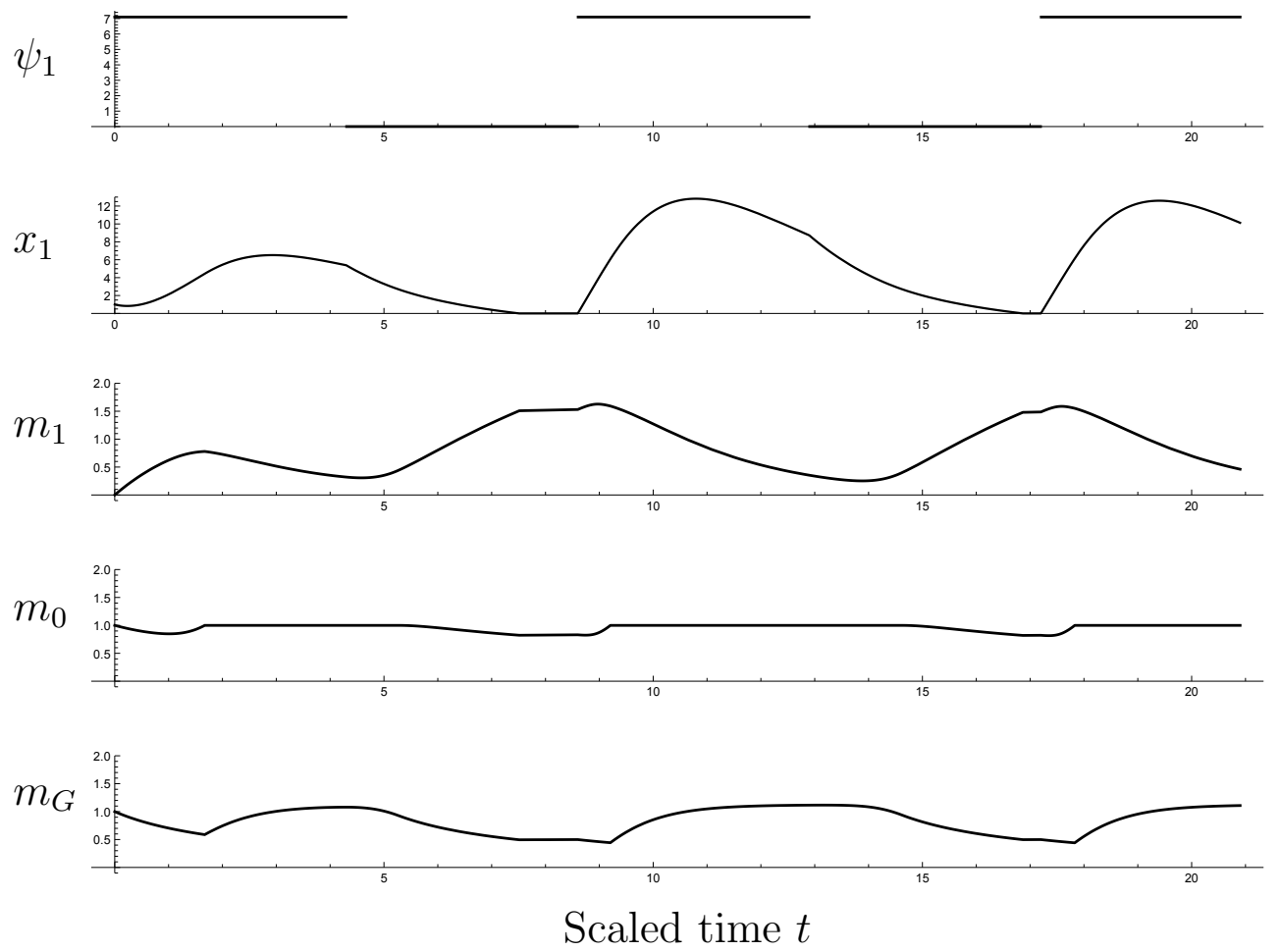

Figure 2. Numerical solutions of the PWS system (24) for $n=1$. The imposed environmental conditions $\psi_{1}(t)$ are shown in the top panel and the remaining panels show the resulting solutions of the PWS system, as indicated by the labels. The function $r_{G}$ is as in eqn (18) with $K=10^{4}$ and $r_{G, \max }=100 ; r_{1}=10 /\left(1+\exp \left(x_{1}-1\right)\right) ; \psi_{W}=$ $0.42 ; \sigma_{1}=0.61 ; c_{1}=0.01 ;$ and $\nu=0.01$.

The numerical solutions presented in Fig. 2 were obtained using Mathematica's built-in tools to detect points in time at which discontinuity occurs, verify that the numerical value of the state at this time point lies within a pre-defined tolerance threshold, and perform either the switch to the adjacent vector field or enter a sliding manifold. This gives a reasonably accurate approximation to the system's trajectory right up to this point and employs these values as initial data for the next time segment. This is known as an event-driven method in which the discontinuous system is solved by treating it as a sequence of continuous systems [33].

\subsection{Filippov solution for multiple reserves}

Generalisation to systems with an arbitrary number of essential nutrients and corresponding reserves is straightfoward. The PWS system has the same form as (24), but the state variable $\mathbf{X}$ is extended to accommodate $n$ reserves rather than just the one. This means that the regions $S^{+}$and $S^{-}$are part of a space of higher dimension and we have to redefine the function $h(\mathbf{X})$ accordingly. Recall that $h$ equals zero if and only if $\mathbf{X} \in \Sigma$ (eqn $(21))$. Let $s(\cdot)$ be defined as follows:

$$
s(\mathbf{X})= \begin{cases}+1, & \text { if } x_{j}>0 \text { for all } j \\ -1, & \text { otherwise }\end{cases}
$$


Then

$$
\Sigma=\left\{\mathbf{X} \in \mathbb{R}^{2 n+2}: h(\mathbf{X})=0\right\}
$$

with

$$
h(\mathbf{X})=s(\mathbf{X}) \min \left(\left|x_{1}\right|, \ldots,\left|x_{n}\right|\right) .
$$

Without serious loss of generality, we may consider only the case where the trajectory attains the boundary at a point where $x_{j}=0$ for exactly one value of $j$, all other cases being non-generic (i.e. corresponding to a subset of initial conditions of measure zero). In the vicinity of such a generic crossing, all $x_{j}$ but one $\left(x_{k}\right.$, say) remain positive and $x_{k}$ attains the value zero. Thus, in the neighbourhood of a generic crossing, we have

$$
h(\mathbf{X})=s(\mathbf{X}) \min \left(\left|x_{1}\right|, \ldots,\left|x_{n}\right|\right) \equiv \min \left(x_{1}, \ldots, x_{n}\right) \equiv x_{k} .
$$

The regions $S^{+}$and $S^{-}$correspond to the sign of the function $h(\mathbf{X})$, as in Section 4.1:

$$
S^{+}=\left\{\mathbf{X} \in \mathbb{R}^{2 n+2}: h(\mathbf{X})>0\right\}, \quad S^{-}=\left\{\mathbf{X} \in \mathbb{R}^{2 n+2}: h(\mathbf{X})<0\right\} .
$$

The dynamic flows in the two domains are

$$
f^{+}(\mathbf{X})=\left(\xi_{j}^{+} ; \eta_{i}^{+}\right)^{T}, \quad f^{-}(\mathbf{X})=\left(\xi_{j}^{-} ; \eta_{i}^{-}\right)^{T}
$$

for $j \in\{1, \ldots, n\}, i \in\{0,1, \ldots, n, G\}$, where

$$
\xi_{j}^{+}=\psi_{j} m_{j}-\psi_{W} m_{G}\left(1+x_{j}\right)-\sigma_{j} m_{0}-c_{j}, \quad \eta_{i}^{+}=\alpha_{i} m_{0}-\psi_{W} m_{G} m_{i}
$$

for $j \in\{1, \ldots, n\}$ and $i \in\{0,1, \ldots, n, G\}$. The vector field in the region $S^{-}$is defined in analogy to eqn (23):

$$
\xi_{j}^{-}=\psi_{j} m_{j}+\nu\left(1+x_{j}\right), \quad \eta_{i}^{-}=\nu m_{i},
$$

where $j \in\{1, \ldots, n\}, i \in\{0,1, \ldots, n, G\}$ and $\nu$ is a positive constant. Calculations entirely analogous to those in Section 4.1 now yield $\lambda=\xi_{k}^{-} /\left(\xi_{k}^{-}-\xi_{k}^{+}\right)$. As before we find that the sliding mode is feasible, by virtue of stationarity on $\Sigma$ :

$$
f^{F}\left(x_{k}\right)=(1-\lambda) \xi_{k}^{-}+\lambda \xi_{k}^{+}=\frac{-\xi_{k}^{+}}{\xi_{k}^{-}-\xi_{k}^{+}} \xi_{k}^{-}+\frac{\xi_{k}^{-}}{\xi_{k}^{-}-\xi_{k}^{+}} \xi_{k}^{+}=0,
$$

where $k$ is the index of the "limiting" reserve (i.e. $x_{k}=0$ ). The arguments presented for $n=1$ in Section 4.1 go through virtually unchanged. The overall behaviour of the solution of system (24) can be described as follows: it starts at an initial position $\mathbf{X}_{0}$ in the subspace $S^{+}$and continues its motion along the vector field $f^{+}(\mathbf{X})$ until the trajectory reaches the surface of discontinuity $\Sigma$ at some point in time, when one of the reserve is depleted $\left(x_{k}=0\right)$, whereas the other reserves can still furnish building blocks $\left(x_{\ell}>0, \ell \neq k\right)$. If the level of the corresponding nutrient $k$ in the environment at this moment in time is such that the corresponding coefficient $\psi_{k}$ is greater than its critical value $\psi_{k}^{\star}=\left(\psi_{W} m_{G}+\sigma_{k} m_{0}+c_{k}\right) / m_{k}$, the solution will exit the boundary and return 
to $S^{+}$. Otherwise, the trajectory enters the sliding mode on the boundary until the level of the nutrient $k$ increases sufficiently to ensure that the inequality $\psi_{k} \geq \psi_{k}^{\star}$ holds true.

\section{Regularisation}

The event-driven method used earlier to obtain numerical solution can be contrasted to the regularisation approach which attempts to approximate the trajectory of the PWS system with that of a system whose vector field is smooth over the entire domain of interest, such that the behaviour of the approximating system can be expected to converge to that of the PWS system as $\epsilon \rightarrow 0^{+}$for some suitable parameter $\epsilon>0$.

In dynamic regularisation [34], an auxiliary state $\mathbf{Z} \in \mathbb{R}^{n}$ is introduced to approximate solutions to the problem as stated, i.e.

$$
\dot{\mathbf{X}}=f(\mathbf{X})=\left\{\begin{array}{ll}
f^{+}(\mathbf{X}), & \text { if } h(\mathbf{X})>0 \\
f^{-}(\mathbf{X}), & \text { if } h(\mathbf{X})<0,
\end{array} \quad \mathbf{X}(0)=\mathbf{X}_{0} \in \mathbb{R}^{n}\right.
$$

by the solutions of the following system of delay-differential equations:

$$
\dot{\mathbf{X}}_{\epsilon}=\mathbf{Z} ; \quad \dot{\mathbf{Z}}=\epsilon^{-1} \nabla_{\epsilon} f\left(\mathbf{X}_{\epsilon}\right) ; \quad \mathbf{X}_{\epsilon}(0)=\mathbf{X}_{0} ; \quad \mathbf{Z}(t)=\boldsymbol{\zeta}(t), \quad t \in[-\epsilon, 0],
$$

where $\nabla_{\epsilon}$ is the backward difference operator with lag $\epsilon$; provided that the continuous function $\boldsymbol{\zeta}(\cdot)$ satisfying certain regularity properties, the approximation $\mathbf{X}_{\epsilon}(t)$ converges to the Filippov solution for $\mathbf{X}(t)$ as $\epsilon \rightarrow 0^{+}$[34].

In flow field regularisation [35-37], the discontinuous vector field $f(\cdot)$ is replaced by a smooth approximation which agrees with the original field except within distance $O(\epsilon)$ of the surface of discontinuity:

$$
\dot{\mathbf{X}}_{\epsilon}=f_{\epsilon}\left(\mathbf{X}_{\epsilon}\right)=\left(1-\theta_{\epsilon}\left(h\left(\mathbf{X}_{\epsilon}\right)\right)\right) f^{-}\left(\mathbf{X}_{\epsilon}\right)+\theta_{\epsilon}\left(h\left(\mathbf{X}_{\epsilon}\right)\right) f^{+}\left(\mathbf{X}_{\epsilon}\right),
$$

where $\theta_{\epsilon}(\cdot)$ is a regularisation function, defined as follows:

$$
\theta_{\epsilon}(h)=\frac{1}{\epsilon} \int_{-\infty}^{h} \rho\left(\frac{s}{\epsilon}\right) d s,
$$

where $\rho(\cdot)$ is a distribution function with support $[-1,1]$. It follows that $\theta_{\epsilon}(h)$ is nondecreasing on the interval $(-\epsilon, \epsilon)$ and agrees with the Heaviside function when $|h| \geq \epsilon$ :

$$
\theta_{\epsilon}(h)=0 \text { if } h \leq-\epsilon ; \quad \theta_{\epsilon}(h)=1 \text { if } h \geq \epsilon .
$$

The approximation $\mathbf{X}_{\epsilon}(t)$ obtained by means of this regularisation converges uniformly to the Filippov solution of system (28) for $\epsilon \rightarrow 0$ [37]. The concatenation of "height" function $h$ and regularisation $\theta_{\epsilon}$ is formally identical to the control input $u$ in eqn (4). Thus, the flow field regularisation approach can be viewed as a continuous approximation to continuous control $[3,4]$. 

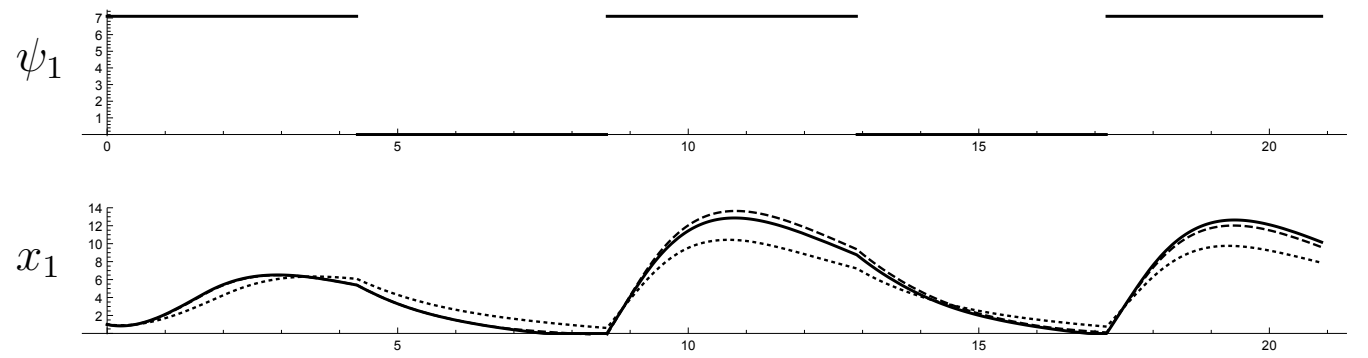

Scaled time $t$

Figure 3. Numerical solutions of system (24) with its space regularisation with piecewise function $\theta$ defined by eqn (35) with parameter $\epsilon=0.1$ for $n=1$. The function $r_{G}$ is as in eqn (18) with $K=10^{4}$ and $r_{G, \max }=$ $100 ; r_{1}=10 /\left(1+\exp \left(x_{1}-1\right)\right) ; \psi_{W}=0.42 ; \sigma_{1}=0.61 ; c_{1}=0.1 ;$ and $\nu=1$. The top panel shows the imposed environmental regime, whereas the bottom panel shows the reserve density for $\epsilon=10$ (dotted line); $\epsilon=1$ (dashed line); and $\epsilon=0.01$ (solid line); the latter is indistinguishable from Mathematica's built-in event-driven method.

\subsection{Biological interpretation of the flow field regularisation}

The regularisation, eqn (30), permits a natural interpretation in terms of the biological system at hand. In particular, let us consider the approximate dynamics of the reserve densities:

$$
\dot{x}_{j, \epsilon}=\psi_{j} m_{j, \epsilon}+\left(1-\theta_{\epsilon}\right) \nu\left(1+x_{j, \epsilon}\right)-\theta_{\epsilon}\left(\psi_{W} m_{G, \epsilon}\left(1+x_{j, \epsilon}\right)+\sigma_{j} m_{0, \epsilon}+c_{j}\right)
$$

and of the machinery densities:

$$
\dot{m}_{i, \epsilon}=\left(1-\theta_{\epsilon}\right) \nu m_{i, \epsilon}+\theta_{\epsilon}\left(\alpha_{i} m_{0, \epsilon}-\psi_{W} m_{G, \epsilon} m_{i, \epsilon}\right) .
$$

In order to relate these equations to a physiologically plausible interpretation, we recall that the switching function $h$, the argument of $\theta_{\epsilon}$, is just the density of the "limiting" reserve, and we consider these equations assuming that (i) we have $\nu \ll 1$ (in keeping with our earlier discussion); and (ii) almost all of the distribution $\rho$ 's probability mass is centered on the interval $[0,1]$. Under these assumptions, we may ignore the terms with $\left(1-\theta_{\epsilon}\right) \nu$ and think of $\theta_{\epsilon}$ as a dimensionless multiplicatory modification of the rates of metabolic expenditure. That latter is made up of fluxes through the various anabolic pathways, together with the endogeneous metabolism that generates the required Gibbs enthalpic driving force. This "rate multiplier" $\theta_{\epsilon}$ has a physiologically sensible behaviour: it remains at the value 1 as long as all reserve densities are bounded away from $\epsilon$ and no metabolic "shut down" is required, and, furthermore, $\theta_{\epsilon}$ traverses the interval $(0,1]$ from above as the limiting reserve density decreases through the value $\epsilon$ and lower. Intuitively, expenditures are "pinched" as the limiting reserve density approaches complete depletion. 


\subsection{Convergence and robustness of the flow field regularisation}

Let us apply the flow field regularisation to system (24) for the case $n=1$. Assuming that

$$
\rho(t)= \begin{cases}1 / 2, & \text { if } t \in[-1,1] \\ 0, & \text { otherwise }\end{cases}
$$

and recalling that $h(\mathbf{X})=x_{1}$, we define the regularisation function $\theta_{\epsilon}$ for system (24) as the following piecewise function:

$$
\theta\left(x_{1}\right)= \begin{cases}0, & \text { if } x_{1} \leq-\epsilon \\ \left(x_{1} / \epsilon+1\right) / 2, & \text { if } x_{1} \in(-\epsilon, \epsilon) \\ 1, & \text { if } x_{1} \geq \epsilon\end{cases}
$$

Numerical solutions of the regularised system (24) by means of the piecewise regularisation function $\theta$ defined by eqn (35) are shown in Fig. 3. For sufficiently small values of $\epsilon$, the regularised numerical solution virtually coincides with the event-driven numerical solution, which, in tandem with the convergence result due to Schiller and Arnold [37], supports our confidence in both. Moreover, even if Mathematica's built-in approximation to Filippov sliding mode solutions is admirably sophisticated, failure to exit the sliding mode occurs intermittently when $\nu$ is set to moderately small values (the intermittency is unpredictable for all intents and purposes and presumably due to round-off error). The regularisation does not suffer from this problem and thus appears to be a superior numerical method for the system at hand.

\section{Discussion}

We have analysed a variable-internal-stores (VIS) model of microbial metabolism and growth that accounts for changes in cellular composition in response to fluctuations in nutrient availability in the environment. The model comprises stoichiometric balance equations together with constitutive equations that describe the adaptive allocation of molecular building blocks among various types of catalytic machinery; it has a unique, stable equilibrium point. Although the model may appear somewhat more involved than the classical models [25, 27, 28, 38-40], it reduces to these models under suitable assumptions [20] and is the simplest mathematical framework in which these classical models can be made consistent with stoichiometric conservation principles and the principles of building block allocation (which in the biological system is effected by the regulatory interactions between transcription and translation); moreover, the formalism presented here is consistent with a mechanism of growth regulation featured in the Scott-Hwamodel [41-43].

We have considered the PWS system as consisting of the dynamics of the original model that deals with intensive variables only, i.e., density variables that do not scale with the overall size of the system (cell size, or, more generally, the size of a colony of cells described by the present model). However, there is one additional extensive variable, $W$. In the region $S^{+}$, we have the identities $\mu=\left(W \widetilde{\phi}_{0}\right)^{-1} \dot{W}=\psi_{W} m_{G}$, whereas $\left(W \widetilde{\phi}_{0}\right)^{-1} \dot{W}=-\nu$ in $S^{-}$. The intensive dynamics allowed us to avoid the question of what happens to the growth rate $\dot{W}$ on $\Sigma$, which we may not answer by noting that it is subject to the convex 
combination prescribed by Filippov:

$$
\frac{\dot{W}}{W \widetilde{\phi}_{0}}=\frac{-\xi_{k}^{+}}{\xi_{k}^{-}-\xi_{k}^{+}}(-\nu)+\frac{\xi_{k}^{-}}{\xi_{k}^{-}-\xi_{k}^{+}} \psi_{W} m_{G}
$$

(cf. eqn (27)) which evaluates to a much reduced relative rate of growth, vanishing in the double limit $\nu \rightarrow 0^{+}, \psi_{k} \rightarrow 0^{+}$(where $k$ is the index of the depleted reserve).

We studied the behaviour of the model under conditions of starvation, and considered, in particular, what happens when at least one of the reserves in the cell approaches a critical level of depletion, which forces the organism to switch to a "survival" mode in which metabolic requirements are minimised. In the model, all reserve densities have to be bounded away from zero for metabolism and growth to proceed at normal rates. As long as this condition is satisfied, it is legitimate to treat the metabolic fluxes as demand-driven, at least on a macrochemical level. The microchemical justification of this assumption essentially resides in the homeostatic maintenance of an intracellular pool of small core metabolites, which are continually being replenished from the reserves, and which feed the anabolic pathways. It is precisely this assumption that breaks down under starvation conditions. Such conditions arise when there is an insufficient supply from the environment to keep the reserves bounded away from zero while they are being depleted at normal rates. In such cases, a supply-side modulation of the rates must be included explicitly in the equations.

Although we have argued here that sliding modes are the natural expression for such a supply-side modification of the model, it is instructive to imagine a theoretical biologist who adopts an approach that perhaps comes more naturally to mathematical modellers, which is to modify the original model outright, along the following lines (we take $n=1$ for the sake of simplicity):

$$
\begin{aligned}
\dot{x}_{1} & =\psi_{1} m_{1}-g\left(x_{1}\right)\left(\psi_{W} m_{G}\left(1+x_{1}\right)+\sigma_{1} m_{0}+c_{1}\right) \\
\dot{m}_{i} & =g\left(x_{1}\right)\left(\alpha_{i} m_{0}-\psi_{W} m_{G} m_{i}\right),
\end{aligned}
$$

where $g(\cdot)$ is a function with the properties $g(0)=0, g^{\prime}(x) \geq 0$, and $g(x) \leq 1 \forall x$; for example

$$
g\left(x_{1}\right)=x_{1} /\left(\varkappa+x_{1}\right)
$$

with positive parameter $\varkappa$. The theoretical biologist views $g(\cdot)$ as a "throttle," reducing metabolic expenditures when the reserve density runs low (cf. Section 5.1). Any such model can be cast as a regularisation by choosing a switching function $h(\cdot)$ and distribution $\rho(\cdot)$ so that the resulting function $\theta_{\epsilon}(\cdot)$ provides a mock-up for the modeller's specification of $g(\cdot)$. Schiller and Arnold's theorem [37] guarantees that there is a corresponding Filippov dynamics that arises as a limiting case, (for eqn (37) this would be the limit $\varkappa \rightarrow 0$ ). This strongly suggests that sliding mode Filippov solutions are a natural, generic mathematical framework in which to express metabolic "shut down."

We suggest that mathematical models of metabolism and biochemical pathways will generically permit such a correspondence between smooth multiplier-type control models and regularisations of PWS systems. This could be exploited to furnish a systematic procedure to derive self-consistent PWS representations of biological processes, which is a non-trivial challenge in general. From the PWS point of view, starting with more complicated smooth dynamics may appear to be an unnecessary detour, but this approach 
is more intuitive and avoids the pitfalls associated with writing down PWS equations directly.

If the flow field regularisation has a counterpart in smooth models with natural biological interpretations, one may wonder if the same can be said for dynamic regularisation. At first blush this might seem a less promising avenue, not only because the required increase in state space dimension, but also because of the lack of an obvious biological counterpart for the backward difference operator $\nabla_{\epsilon}$ that appears in eqn (29). The latter objection is somewhat alleviated by appealing to the "linear chain trick" which can be regarded as a soft version of the hard nonlinear delay, and which can be justified as a model of a multi-component signalling cascade [44].

A hybrid of the field and dynamic regularisations was proposed previously to model metabolic "shut down," involving an auxiliary variable, as in the dynamic regularisation, but letting it act as a modulatory multiplier, as in flow field regularisation [25]. By way of definite example, let us replace $\theta_{\epsilon}$ by a new state variable variable $z(t)$ that obeys the following ODE:

$$
\dot{z}=\varsigma z\left((1+\epsilon / h)^{-1}-z\right),
$$

where $\varsigma>0$ sets the time scale of $z$ 's dynamics and $\epsilon>0$ is, as before, a small parameter that governs the width of the transition layer near $\Sigma$. This approach can be extended considerably by having a dedicated dynamic modulatory multiplier for every individual metabolic flux or process, rather than a single one that controls the overall rate of metabolic "shut down." An extension of this form has been proposed as a generic approach to organismal homeostasis [45].

\section{Funding}

O. A. Nev was supported by EU Research Framework programme 7 Marie Curie Actions, grant 316630, Centre for Analytical Science Innovative Doctoral Programme (CAS-IDP).

\section{References}

[1] Dieci L, Lopez L. Sliding motion in Filippov differential systems: theoretical results and a computational approach. SIAM J Numer Anal. 2009;47.

[2] Filippov AF. Differential Equations with Discontinuous Right-Hand Side. Kluwer Academic; 1988.

[3] Utkin VI. Sliding Modes in Control and Optimization. Springer-Verlag; 1992.

[4] Utkin VI. Variable structure systems with sliding modes. IEEE Trans Autom Control. 1977; $22: 212-222$.

[5] Schumacher JM, Weiland S, Heemels WPMH. Linear complementarity systems. SIAM J Appl Math. 2000;60:1234-1269.

[6] Malmborg J, Bernhardsson B. Control and simulation of hybrid systems. Commun Nonlinear Sci. 1997;30:337-347.

[7] Chong EKP, Hui S, Zak SH. An analysis of a class of neural networks for solving linear programming problems. IEEE Trans Autom Control. 1999;44:1995-2006.

[8] Johansson KH, Barabanov AE, Astrom KJ. Limit cycles with chattering in relay feedback systems. IEEE Trans Autom Control. 2002;247:1414-1423.

[9] Johansson KH, Rantzer A, Astrom KJ. Fast switches in relay feedback systems. Automatica J IFAC. 1999;35:539-552. 
[10] Glass L, Kauffman SA. The logical analysis of continuous non-linear biochemical control networks. J Theor Biol. 1973;39:103-129.

[11] Thomas R, Thieffry D, Kaufman M. Dynamical behaviour of biological regulatory networks: I. Biological role of feedback loops and practical use of the concept of the loop-characteristic state. Bull Math Biol. 1995;57:247-276.

[12] Casey R, de Jong H, Gouze JL. Piecewise-linear models of genetics regulatory networks: Equilibria and their stability. J Math Biol. 2006;52:27-56.

[13] de Jong H, Gouze JL, Hernandez C, Page M, Sar T, Geiselmann J. Qualitative simulation of genetic regulatory networks using piecewise-linear models. Bull Math Biol. 2004;66:301-340.

[14] Plahte E, Kjoglum S. Analysis and genetic properties of gene regulatory networks with graded response functions. Physica D. 2005;201:150-176.

[15] Gouze JL, Sari T. A class of piecewise linear differential equations arising in biological models. Dyn Syst. 2002;17:299-316.

[16] Williams FM. A model of cell growth dynamics. J Theor Biol. 1967;15:190-207.

[17] Droop MR. Vitamin B12 and marine ecology. IV. The kinetics of uptake, growth and inhibition in Monochrysis lutheri. J Mar Biol Assoc. 1968;48.

[18] Grover JP. Resource competition in a variable environment: Phytoplankton growing according to the Variable-Internal-Stores model. Am Nat. 1991;138:811-835.

[19] Dawes EA. Growth and survival of bacteria. In: Poindexter JS, Leadbetter ER, editors. Bacteria in nature III: Structure, physiology, and genetic adaptability. Plenum; 1989. p. 67-187.

[20] Nev OA, van den Berg HA. Variable-Internal-Stores models of microbial growth and metabolism with dynamic allocation of cellular resources. J Math Biol. 2016; DOI: $10.1007 / \mathrm{s} 00285-016-1030-4$.

[21] Bleecken S. Model for the feedback control system of bacterial growth I. Growth in discontinuous culture. J Theor Biol. 1988;133:37-65.

[22] van den Berg HA. How microbes can achieve balanced growth in a fluctuating environment. Acta Biotheor. 2001;49:1-21.

[23] Kramer G, Sprenger RR, Nessen MA, Roseboom W, Speijer D, de Jong L, Texeira de Mattos MJ, Back J, de Koster CG. Proteome-wide alterations in Escherichia coli translation rates upon anaerobiosis. Mol Cell Proteomics. 2010;9.11:2508-2516.

[24] Neidhardt FC, Ingraham JL, Schaechter M. Physiology of the bacterial cell: A molecular approach. Sinauer Associates; 1990.

[25] van den Berg HA. A generic view of classic microbial growth models. Acta Biotheor. 1998; 46:117-130.

[26] Aubin JP, Cellina A. Differential Inclusions. Springer-Verlag; 1984.

[27] Marr AG, Nilson EH, Clark DJ. The maintenance requirement of Escherichia coli. Ann N Y Acad Sci. 1962;102:536-548.

[28] Pirt SJ. The maintenance energy of bacteria in growing cultures. Proc Roy Soc Lond. 1965; 133:300-302.

[29] Herbert D. The chemical composition of micro-organisms as a function of their environment. Symp Soc Gen Microbiol. 1961;11:391-416.

[30] Broughton C, van den Berg HA, Wemyss AM, Roper DI, Rodger A. Beyond the discovery void: New targets for antibacterial compounds. Science Progress. 2016;99:153-182.

[31] Kowalczyk P, Piiroinen PT. Two-parameter sliding bifurcations of periodic solutions in a dry-friction oscillator. Physica D. 2008;237:1053-1073.

[32] Jeffrey MR, Hogan SJ. The geometry of generic sliding bifurcations. SIAM Rev. 2011;53:505525.

[33] Acary V, Brogliato B. Numerical Methods for Nonsmooth Dynamical Systems. Applications in Mechanics and Electronics. Lecture Notes In Applied and Computational Mechanics. Springer-Verlag; 2008.

[34] Fusco G, Guglielmi N. A regularization for discontinuous differential equations with application to state-dependent delay differential equations of neutral type. J Diff Equations. 2011; 250. 
[35] Sotomayor J, Teixeira MA. Regularization of discontinuous vector fields. In: International Conference on Differential Equations; 1995. p. 207-223.

[36] Sotomayor J, Machado ALF. Structurally stable discontinuous vector fields in the plane. Qual Theory Dyn Syst. 2002;3(1).

[37] Schiller H, Arnold M. Convergence of continuous approximation for discontinuous ODEs. Appl Numer Math. 2012;62(10).

[38] Monod J. The growth of bacterial cultures. Annu Rev Microbiol. 1949;3:371-394.

[39] Droop MR. Vitamin B12 and marine ecology. IV. The kinetics of uptake, growth and inhibition in Monochrysis lutheri. J Mar Biol Assoc. 1968;48:689-733.

[40] Kooijman SALM. Dynamic energy budget theory for metabolic organisation. Cambridge University Press; 2009.

[41] Scott M, Gunderson CW, Mateescu EM, Zhang Z, Hwa T. Interdependence of cell growth and gene expression: Origins and consequences. Science. 2010;330:1099-1102.

[42] Scott M, Hwa T. Bacterial growth laws and their applications. Curr Opinion Biotechnol. 2011;22:599-565.

[43] Scott M, Klumpp S, Mateescu EM, Hwa T. Emergence of robust growth laws from optimal regulation of ribosome synthesis. Mol Syst Biol. 2014;10:747.

[44] Fall CP, Marland ES, Wagner JM, Tyson JJ. Computational cell biology. Springer-Verlag; 2002.

[45] van den Berg HA, Kiselev YN, Orlov MV. Homeostatic regulation in physiological systems: A versatile Ansatz. Math Biosci. 2015;268:92-101. 